\title{
Growth and Yield Characteristics of Capsicum (Capsicum annuum L.) cv. Orobelle in Response to Different Growing Media and Plant Spacing under Protected Conditions
}

\author{
Bijeta*, Kuldeep Singh Thakur, Sandeep Kansal and Ashwini Kumar Sharma
}

Department of Vegetable Science, Dr. Y.S. Parmar University of Horticulture and Forestry, Nauni, Solan, Himachal Pradesh, India

*Corresponding author

\section{A B S T R A C T}

\begin{tabular}{|l|}
\hline K e y w o r d s \\
Plant spacing, Capsicum, \\
Growing media
\end{tabular}

\section{Introduction}

Varied agro ecological conditions offer vast scope for production of vegetables, fruits and other horticultural commodities in Himalayan regions. In general, profits are higher in off season vegetable crops provided climatic constraints are overcome by adopting protected cultivation technologies (Bisht 2012). The purpose of growing crops under greenhouse conditions is to extend cropping season and to protect them from adverse environmental conditions (Sharma et al., 2010, Jaipaul et al., 2011). In India, protected cultivation is gaining momentum in expansion of area and productivity of horticultural crops in a faster rate. Among various vegetable crops, capsicum fits best in summer seasons as offseason crop. Capsicum is an important commercial vegetable crop of mid and high Himalayan region and gives maximum profit to farmers during early and off-season. It is considered to be an export potential commodity. Generally, crop requires warm temperature ranging from 20 to $25^{\circ} \mathrm{C}$ for its growth and development. Especially high hills and mid areas are not considered suitable for open field cultivation. In natural ventilated conditions, capsicum can be cultivated throughout the year except 2-3 months in winters. However, inclement weather accompanied by fluctuating temperature, which is a common feature in hilly regions during rainy season, affects the productivity 
and quality of the produce of this crop when grown under open field conditions and finally reduces the profit margin of the producers. Under such conditions, greenhouse cultivation of capsicum (Sweet pepper) can play an important role in producing and sustaining higher yield and better quality of fruits than the open field cultivation. Greenhouse technology for vegetable production, especially at high altitudes needs to be further exploited and expanded for commercial cultivation of high quality vegetables. Greenhouse provides protection against biotic and abiotic stresses and ensures high quality produce.

The consistent and discriminate use of inorganic fertilizers have caused serious damage to soil health, ecology and caused decline in vitamin and mineral content of fresh fruits and vegetables. The addition of farm organic wastes, manures, cocopeat and vermicompost etc. are extremely important to maintain the fertility and productivity of the agricultural system. Organically grown greenhouse crops in general, have higher nutrient demands than field grown crops and therefore, in order to optimize production it is essential to focus on the growing media studies.

\section{Materials and Methods}

The experiment was carried out in naturally ventilated polyhouse at Vegetable Research Farm of Department of Vegetable Science, Dr. Y. S. Parmar University of Horticulture and Forestry, Nauni, Solan, Himachal Pradesh. Planting of capsicum cv. "Orobelle" was done in Randomized Block Design (Factorial) with three replications during the February 2015 and 2016 inside a naturally ventilated polyhouse. The different growing media in combination with different plant spacings were allotted randomly to the plots. The treatments comprised of four different growing media $(\mathrm{M})$ viz., $\left(\mathrm{M}_{1}\right)$ Soil + Sand + FYM (2:1:1), $\left(\mathrm{M}_{2}\right)$ Soil + Cocopeat + FYM $(2: 1: 1), \quad\left(\mathrm{M}_{3}\right)$ Soil + Cocopeat + Vermicompost $(2: 1: 1),\left(\mathrm{M}_{4}\right)$ Soil + Cocopeat + Vermicompost + FYM (2:1:0.5:0.5) and three plant spacings with different population (S) viz., $45 \times 30 \mathrm{~cm}\left(\mathrm{~S}_{1}\right), 45 \times 45 \mathrm{~cm}\left(\mathrm{~S}_{2}\right)$, and $45 \times 60 \mathrm{~cm}\left(\mathrm{~S}_{3}\right)$. Twelve different Treatment combinations viz., $\mathrm{M}_{1} \mathrm{~S}_{1}, \mathrm{M}_{1} \mathrm{~S}_{2}, \mathrm{M}_{1} \mathrm{~S}_{3}, \mathrm{M}_{2} \mathrm{~S}_{1}$, $M_{2} S_{2}, M_{2} S_{3}, M_{3} S_{1}, M_{3} S_{2}, M_{3} S_{3}, M_{4} S_{1}, M_{4} S_{2}$, $\mathrm{M}_{4} \mathrm{~S}_{3}$ were transplanted in the polyhouse in three replications on $15^{\text {th }}$ April, 2015 and 2016 in a plot having size of $1.62 \mathrm{~m}^{2}$. All the standard recommended cultural practices were followed to raise a successful crop during the course of investigation. Treatment details are given in the Table 1.

Data for growth and yield attributes were recorded on five randomly chosen plants in each treatment. Then the average was calculated. All the growth characters such as Days to 50 per cent flowering, days to marketable maturity, harvest duration (days), and plant height $(\mathrm{cm})$, number of fruits per plant, fruit length $(\mathrm{cm})$, fruit breadth $(\mathrm{cm})$, average fruit weight $(\mathrm{g})$, fruit yield $(\mathrm{kg} / \mathrm{plant}$ and $\mathrm{kg} / \mathrm{m}^{2}$ ), were recorded. The cumulative value of the yield per plant and per $\mathrm{m}^{2}$ was taken as and when the pickings progressed.

\section{Results and Discussion}

\section{Growth parameters}

The data in general revealed that the media consisting of cocopeat, vermicompost and FYM recorded the maximum growth among all the treatments. Perusal of the Table 2 and 3 showed that the minimum values for Days to 50 per cent flowering (41.89) and days to marketable maturity (71.56), whereas, maximum values for harvest duration (85.67), and plant height $(154.46 \mathrm{~cm})$ were recorded in $\mathrm{M}_{4}$ over other growing media during both the years of experimentation. The media $\mathrm{M}_{3}$ 
performed closely behind it and gave the second best results and many times was statistically at par to the highest readings. These results are in accordance to the studies of Roy et al., (2011). They had inferred that vermicompost alone and admixed with FYM and green manure, were found to be effective in improving various plant growth parameters in capsicum. Organic material can be broken down and fragmented rapidly by earthworm, resulting in a stable nontoxic material with appropriative structure which has potentially high economic value as a soil conditioner for plant growth (Hala et al., 2003). Organic growing media have a property of good water holding capacity and are also able to drain excess water to come to field capacity which creates congenial root environment. Considering the results, it is noticed that growth characters of capsicum were increased with application of cocopeat, vermicompost and FYM. These results may be attributed to the role of macro and micro-nutrients, as well as the improved soil conditions due to vermicompost application, which conduced to stimulate metabolic processes and encourage growth, synthesis and accumulation of more metabolites in plant tissues. Several investigators mentioned similar results on different plants such as Kumar and Kohli (2005) in capsicum, Natarajan (2005) in tomato, Bairwa et al., (2009) in Okra. Plant spacing had a significant influence on growth characters of capsicum during both the years. Capsicum raised at $45 \times 60 \mathrm{~cm}\left(\mathrm{~S}_{3}\right)$ obtained minimum days to 50 per cent flowering, minimum (42.29) days to marketable maturity (71.50), taller plants (151.66) and maximum harvest duration (82.71), which was statistically at par with $45 \times 45 \mathrm{~cm}\left(\mathrm{~S}_{2}\right)$. Taller plants might be due to competition of light under $S_{1}(45 \times 30 \mathrm{~cm})$ with closer plant to plant space for want of light. Wider plant spacing had minimum days to 50 per cent flowering, days to marketable maturity, taller plants and highest harvest duration due to better availability of resources. Wider space availability between the plants might have increased the root spread which eventually utilized the resources such as water, nutrients, space and light very effectively. Similar results were reported by Ganjare et al., (2013), Malik et al., (2011), Roy et al., (2011), and Kumar (2011).

\section{Yield parameters}

It is evident from Table 4 that the yield per plant increased significantly with the incorporation of cocopeat and vermicompost together in $\mathrm{M}_{3}$ media. The media consisting of cocopeat, vermicompost and FYM which was followed by $M_{4} . M_{3}$ recorded the highest number of fruits per plant (14.86) and fruit breadth $(9.80 \mathrm{~cm})$. The media $\mathrm{M}_{4}$ recorded the maximum fruit length $(10.25 \mathrm{~cm})$, average fruit weight $(192.91 \mathrm{~g})$, fruit yield (2.81 $\mathrm{kg} /$ plant), whereas $\mathbf{M}_{1}$ gave the poorest yield parameters during both the years. The treatments combining both cocopeat, vermicompost and FYM showed highest yields, while the yields of treatments using cocopeat and vermicompost were also comparable but were significantly lesser than the former. Both performed significantly better than the $M_{1}$. This result is in agreement of the findings of Llaven et al., (2008) in bell pepper, Uma Maheshwari and Haripriya (2007) in hot pepper. The vermicompost based $M_{3}$ and $M_{4}$ media again proved to be better to others as it produced maximum yield parameters followed by $\mathrm{M}_{2}$ and $\mathrm{M}_{1}$ growing media. Wider spacing produced significantly more number of fruits per plant (14.39), fruit length $(9.84 \mathrm{~cm})$, fruit breadth $(9.48 \mathrm{~cm})$, average fruit weight $(227.00 \mathrm{~g})$, fruit yield (3.28 kg/plant) during both the years. The wider spacing leads to more growing area and better competition among plants and subsequently better growth which in turn had a positive effect on yield attributes. Similar results were recorded by Mantur et al., (2005). 
Table.1 Treatment details of the experiment along with symbols

\begin{tabular}{|c|c|c|c|}
\hline Treatment No. & Media & Spacing & Symbol \\
\hline 1. & Soil + Sand + FYM (2:1:1) & $45 \times 30$ & $\mathrm{M}_{1} \mathrm{~S}_{1}$ \\
\hline 2. & Soil + Sand + FYM $(2: 1: 1)$ & $45 \times 45$ & $\mathrm{M}_{1} \mathrm{~S}_{2}$ \\
\hline 3. & Soil + Sand + FYM $(2: 1: 1)$ & $45 \times 60$ & $\mathrm{M}_{1} \mathrm{~S}_{3}$ \\
\hline 4. & Soil + Cocopeat + FYM $(2: 1: 1)$ & $45 \times 30$ & $\mathrm{M}_{2} \mathrm{~S}_{1}$ \\
\hline 5. & Soil + Cocopeat + FYM (2:1:1) & $45 \times 45$ & $\mathrm{M}_{2} \mathrm{~S}_{2}$ \\
\hline 6. & Soil + Cocopeat + FYM $(2: 1: 1)$ & $45 \times 60$ & $\mathrm{M}_{2} \mathrm{~S}_{3}$ \\
\hline 7. & Soil + Cocopeat + Vermicompost $(2: 1: 1)$ & $45 \times 30$ & $\mathrm{M}_{3} \mathrm{~S}_{1}$ \\
\hline 8. & Soil + Cocopeat + Vermicompost $(2: 1: 1)$ & $45 \times 45$ & $\mathrm{M}_{3} \mathrm{~S}_{2}$ \\
\hline 9. & Soil + Cocopeat + Vermicompost $(2: 1: 1)$ & $45 \times 60$ & $\mathrm{M}_{3} \mathrm{~S}_{3}$ \\
\hline 10. & Soil + Cocopeat + Vermicompost + FYM (2:1:0.5:0.5) & $45 \times 30$ & $\mathrm{M}_{4} \mathrm{~S}_{1}$ \\
\hline 11. & Soil + Cocopeat + Vermicompost + FYM (2:1:0.5:0.5) & $45 \times 45$ & $\mathrm{M}_{4} \mathrm{~S}_{2}$ \\
\hline 12. & Soil + Cocopeat + Vermicompost + FYM (2:1:0.5:0.5) & $45 \times 60$ & $\mathrm{M}_{4} \mathrm{~S}_{3}$ \\
\hline
\end{tabular}

Table.2 Days to 50 per cent flowering, days to marketable maturity, harvest duration and plant height, as influenced by growing media, plant spacing and their interaction

\begin{tabular}{|c|c|c|c|c|c|c|c|c|c|c|c|c|}
\hline \multirow[t]{2}{*}{$\begin{array}{c}\text { Treatment } \\
\text { s }\end{array}$} & \multicolumn{3}{|c|}{$\begin{array}{c}\text { Days to } 50 \text { per cent } \\
\text { flowering }\end{array}$} & \multicolumn{3}{|c|}{$\begin{array}{c}\text { Days to marketable } \\
\text { maturity }\end{array}$} & \multicolumn{3}{|c|}{$\begin{array}{l}\text { Harvest duration } \\
\text { (days) }\end{array}$} & \multicolumn{3}{|c|}{ Plant height (cm) } \\
\hline & 2015 & 2016 & 2015 & 2016 & 2015 & 2016 & 2015 & 2016 & Pooled & 2015 & 2016 & Pooled \\
\hline$M_{1}$ & 43.00 & 46.44 & 43.00 & 46.44 & 43.00 & 46.44 & 73.00 & 69.44 & 71.22 & 145.07 & 122.14 & 133.61 \\
\hline $\mathbf{M}_{2}$ & 41.67 & 44.56 & 41.67 & 44.56 & 41.67 & 44.56 & 82.00 & 73.67 & 77.83 & 150.24 & 138.13 & 144.19 \\
\hline $\mathbf{M}_{3}$ & 42.00 & 43.22 & 42.00 & 43.22 & 42.00 & 43.22 & 89.11 & 82.00 & 85.56 & 156.19 & 149.51 & 150.89 \\
\hline $\mathbf{M}_{4}$ & 41.44 & 42.33 & 41.44 & 42.33 & 41.44 & 42.33 & 90.11 & 81.22 & 85.67 & 158.76 & 150.17 & 154.46 \\
\hline Mean & 42.03 & 44.14 & 42.03 & 44.14 & 42.03 & 44.14 & 83.55 & 76.58 & 80.07 & 152.57 & 139.99 & 146.79 \\
\hline $\mathrm{CD}_{0.05}$ & 0.81 & 1.24 & 0.81 & 1.24 & 0.81 & 1.24 & 1.60 & 1.01 & 1.34 & 3.47 & 5.11 & 4.37 \\
\hline$\frac{S_{1}}{4}$ & 42.25 & 44.92 & 42.25 & 44.92 & 42.25 & 44.92 & 79.08 & 72.00 & 75.54 & 142.28 & 132.48 & 137.38 \\
\hline $\mathrm{S}_{2}$ & 42.42 & 44.25 & 42.42 & 44.25 & 42.42 & 44.25 & 85.25 & 78.67 & 81.96 & 157.42 & 142.17 & 148.33 \\
\hline $\mathrm{S}_{3}$ & 41.42 & 43.25 & 41.42 & 43.25 & 41.42 & 43.25 & 86.33 & 79.08 & 82.71 & 158.00 & 145.31 & 151.66 \\
\hline Mean & 42.03 & 44.14 & 42.03 & 44.14 & 42.03 & 44.14 & 83.55 & 76.58 & 80.07 & 152.57 & 139.99 & 146.79 \\
\hline $\mathrm{CD}_{0.05}$ & 0.71 & 1.07 & 0.71 & 1.07 & 0.71 & 1.07 & 1.39 & 0.87 & 1.16 & 3.00 & 4.43 & 3.78 \\
\hline \multicolumn{13}{|l|}{ Interaction : } \\
\hline $\mathbf{M}_{1} \mathbf{S}_{1}$ & 43.00 & 48.33 & 45.67 & 72.67 & 74.67 & 73.67 & 70.00 & 65.00 & 67.50 & 132.40 & 115.00 & 123.70 \\
\hline $\mathbf{M}_{1} \mathbf{S}_{2}$ & 44.00 & 45.67 & 44.83 & 72.33 & 74.00 & 73.17 & 77.00 & 72.33 & 74.67 & 154.67 & 122.17 & 138.42 \\
\hline $\mathbf{M}_{1} \mathbf{S}_{3}$ & 42.00 & 45.33 & 43.67 & 72.00 & 73.67 & 72.83 & 72.00 & 71.00 & 71.50 & 148.15 & 129.27 & 138.71 \\
\hline $\mathrm{M}_{2} \mathrm{~S}_{1}$ & 42.33 & 44.33 & 43.33 & 72.33 & 73.67 & 73.00 & 72.00 & 67.33 & 69.67 & 136.40 & 122.32 & 129.36 \\
\hline $\mathrm{M}_{2} \mathrm{~S}_{2}$ & 41.00 & 45.00 & 43.00 & 71.00 & 72.33 & 71.67 & 85.00 & 75.33 & 80.17 & 156.67 & 143.35 & 150.01 \\
\hline $\mathrm{M}_{2} \mathrm{~S}_{3}$ & 41.67 & 44.33 & 43.00 & 70.67 & 72.00 & 71.33 & 89.00 & 78.33 & 83.67 & 157.67 & 148.72 & 153.19 \\
\hline $\mathbf{M}_{3} \mathbf{S}_{1}$ & 42.33 & 44.00 & 43.17 & 71.67 & 73.33 & 72.50 & 86.33 & 80.00 & 83.17 & 150.13 & 146.85 & 148.49 \\
\hline $\mathbf{M}_{3} \mathbf{S}_{2}$ & 42.67 & 43.67 & 43.17 & 71.33 & 72.67 & 72.00 & 89.00 & 82.33 & 85.67 & 157.59 & 150.75 & 148.29 \\
\hline $\mathbf{M}_{3} \mathbf{S}_{3}$ & 41.00 & 42.00 & 41.33 & 70.67 & 71.67 & 71.17 & 92.00 & 83.67 & 87.83 & 160.84 & 150.94 & 155.89 \\
\hline $\mathbf{M}_{4} \mathbf{S}_{1}$ & 41.33 & 43.00 & 42.17 & 72.00 & 73.33 & 72.67 & 88.00 & 75.67 & 81.83 & 150.17 & 145.75 & 147.96 \\
\hline $\mathbf{M}_{4} \mathbf{S}_{2}$ & 42.00 & 42.67 & 42.33 & 71.00 & 71.67 & 71.33 & 90.00 & 84.67 & 87.33 & 160.77 & 152.42 & 156.59 \\
\hline $\mathbf{M}_{4} \mathbf{S}_{3}$ & 41.00 & 41.33 & 41.17 & 70.00 & 71.33 & 70.67 & 92.33 & 83.33 & 87.83 & 165.34 & 152.33 & 158.83 \\
\hline Mean & 42.03 & 44.14 & 43.07 & 71.47 & 72.86 & 72.17 & 83.55 & 76.58 & 80.07 & 152.57 & 139.99 & 146.79 \\
\hline $\mathrm{CD}_{0.05}$ & NS & NS & NS & NS & NS & NS & 2.78 & 1.74 & 2.32 & 6.00 & 8.85 & 7.56 \\
\hline
\end{tabular}


Table.3 No. of fruits per plant, average fruit weight, fruit length, fruit breadth as influenced by growing media, plant spacing and their interaction

\begin{tabular}{|c|c|c|c|c|c|c|c|c|c|c|c|c|}
\hline \multirow[t]{2}{*}{ Treatments } & \multicolumn{3}{|c|}{ No. of fruits per plant } & \multicolumn{3}{|c|}{ Average fruit weight (g) } & \multicolumn{3}{|c|}{ Fruit length $(\mathrm{cm})$} & \multicolumn{3}{|c|}{ Fruit breadth (cm) } \\
\hline & 2015 & 2016 & Pooled & 2015 & 2016 & Pooled & 2015 & 2016 & Pooled & 2015 & 2016 & Pooled \\
\hline $\mathbf{M}_{1}$ & 12.02 & 11.60 & 11.81 & 149.48 & 148.46 & 148.97 & 8.62 & 8.33 & 8.48 & 7.86 & 7.96 & 7.91 \\
\hline $\mathbf{M}_{2}$ & 13.91 & 13.01 & 13.46 & 163.93 & 157.20 & 160.57 & 9.53 & 8.93 & 9.23 & 9.04 & 8.58 & 8.81 \\
\hline $\mathbf{M}_{3}$ & 15.41 & 14.32 & 14.86 & 180.54 & 190.00 & 185.27 & 10.13 & 10.18 & 10.15 & 9.73 & 9.87 & 9.80 \\
\hline $\mathbf{M}_{4}$ & 15.00 & 13.96 & 14.48 & 188.72 & 197.10 & 192.91 & 10.28 & 10.21 & 10.25 & 9.77 & 9.66 & 9.72 \\
\hline Mean & 14.08 & 13.22 & 13.65 & 171.67 & 173.19 & 171.86 & 9.63 & 9.41 & 9.53 & 9.11 & 9.02 & 9.06 \\
\hline $\mathrm{CD}_{0.05}$ & 0.69 & 0.63 & 0.66 & 9.09 & 10.74 & 9.95 & 0.29 & 0.15 & 0.23 & 0.28 & 0.17 & 0.23 \\
\hline$S_{1}$ & 13.42 & 12.52 & 12.97 & 121.62 & 120.94 & 121.09 & 9.07 & 8.78 & 8.93 & 8.41 & 8.20 & 8.30 \\
\hline $\mathrm{S}_{2}$ & 13.95 & 13.25 & 13.60 & 165.84 & 169.16 & 167.50 & 9.89 & 9.72 & 9.81 & 9.38 & 9.42 & 9.40 \\
\hline $\mathrm{S}_{3}$ & 14.88 & 13.90 & 14.39 & 224.54 & 229.47 & 227.00 & 9.94 & 9.74 & 9.84 & 9.53 & 9.43 & 9.48 \\
\hline Mean & 14.08 & 13.22 & 13.65 & 171.67 & 173.19 & 171.86 & 9.63 & 9.41 & 9.53 & 9.11 & 9.02 & 9.06 \\
\hline $\mathrm{CD}_{0.05}$ & 0.57 & 0.54 & 0.57 & 7.87 & 9.30 & 8.61 & 0.25 & 0.13 & 0.20 & 0.25 & 0.15 & 0.20 \\
\hline \multicolumn{13}{|l|}{ Interaction } \\
\hline $\mathbf{M}_{1} \mathbf{S}_{1}$ & 10.67 & 10.25 & 10.46 & 117.49 & 115.97 & 116.73 & 8.40 & 8.00 & 8.20 & 7.43 & 7.52 & 7.47 \\
\hline $\mathbf{M}_{1} \mathbf{S}_{2}$ & 12.50 & 12.25 & 12.38 & 135.31 & 136.73 & 136.02 & 8.71 & 8.75 & 8.73 & 8.32 & 8.50 & 8.41 \\
\hline $\mathbf{M}_{1} \mathbf{S}_{3}$ & 12.90 & 12.30 & 12.60 & 195.64 & 192.67 & 194.16 & 8.74 & 8.25 & 8.50 & 7.84 & 7.87 & 7.86 \\
\hline $\mathbf{M}_{2} \mathbf{S}_{1}$ & 13.00 & 11.98 & 12.49 & 120.32 & 117.55 & 118.94 & 8.76 & 8.25 & 8.51 & 8.34 & 7.77 & 8.06 \\
\hline $\mathbf{M}_{2} \mathbf{S}_{2}$ & 13.32 & 12.64 & 12.98 & 165.27 & 156.02 & 160.65 & 9.92 & 9.03 & 9.48 & 9.58 & 8.75 & 9.17 \\
\hline $\mathrm{M}_{2} \mathrm{~S}_{3}$ & 15.42 & 14.41 & 14.91 & 206.21 & 198.03 & 202.12 & 9.90 & 9.50 & 9.70 & 9.20 & 9.22 & 9.21 \\
\hline $\mathbf{M}_{3} \mathbf{S}_{1}$ & 15.27 & 14.26 & 14.77 & 121.22 & 122.02 & 121.62 & 9.43 & 9.45 & 9.44 & 9.03 & 9.15 & 9.09 \\
\hline$M_{3} S_{2}$ & 15.32 & 14.39 & 14.85 & 173.52 & 183.36 & 178.44 & 10.42 & 10.50 & 10.46 & 9.83 & 10.20 & 10.02 \\
\hline $\mathbf{M}_{3} \mathbf{S}_{3}$ & 15.63 & 14.30 & 14.97 & 246.86 & 264.62 & 255.74 & 10.53 & 10.58 & 10.56 & 10.34 & 10.25 & 10.30 \\
\hline $\mathbf{M}_{4} \mathbf{S}_{1}$ & 14.75 & 13.57 & 14.16 & 127.45 & 128.21 & 127.83 & 9.70 & 9.43 & 9.57 & 8.84 & 8.36 & 8.60 \\
\hline $\mathbf{M}_{4} \mathbf{S}_{2}$ & 14.67 & 13.73 & 14.20 & 189.25 & 200.53 & 194.89 & 10.53 & 10.59 & 10.56 & 9.77 & 10.22 & 9.99 \\
\hline $\mathbf{M}_{4} \mathbf{S}_{3}$ & 15.58 & 14.59 & 15.09 & 249.44 & 262.57 & 256.01 & 10.60 & 10.62 & 10.61 & 10.72 & 10.40 & 10.56 \\
\hline Mean & 14.08 & 13.22 & 13.65 & 171.67 & 173.19 & 171.86 & 9.63 & 9.41 & 9.53 & 9.11 & 9.02 & 9.06 \\
\hline $\mathrm{CD}_{0.05}$ & 1.14 & 1.08 & 1.14 & 15.74 & 18.60 & 17.23 & NS & 0.26 & 0.40 & 0.49 & 0.29 & 0.40 \\
\hline
\end{tabular}


Table.4 Yield of sweet pepper as influenced by growing media, plant spacing and their interaction

\begin{tabular}{|c|c|c|c|c|c|c|}
\hline \multirow[t]{2}{*}{ Treatments } & \multicolumn{3}{|c|}{ Fruit yield (kg/plant) } & \multicolumn{3}{|c|}{ Fruit yield $\left(\mathrm{kg} / \mathrm{m}^{2}\right)$} \\
\hline & 2015 & 2016 & Pooled & 2015 & 2016 & Pooled \\
\hline $\mathbf{M}_{1}$ & 1.82 & 1.74 & 1.78 & 10.07 & 9.66 & 9.86 \\
\hline $\mathbf{M}_{2}$ & 2.31 & 2.07 & 2.19 & 12.78 & 11.47 & 12.13 \\
\hline $\mathbf{M}_{3}$ & 2.79 & 2.72 & 2.75 & 15.38 & 14.94 & 15.16 \\
\hline $\mathbf{M}_{4}$ & 2.85 & 2.77 & 2.81 & 15.74 & 15.24 & 15.49 \\
\hline Mean & 2.44 & 2.33 & 2.38 & 13.49 & 12.83 & 13.16 \\
\hline $\mathrm{CD}_{0.05}$ & 0.09 & 0.09 & 0.09 & 0.59 & 0.49 & 0.54 \\
\hline $\mathrm{S}_{1}$ & 1.64 & 1.52 & 1.58 & 13.08 & 12.11 & 12.59 \\
\hline $\mathrm{S}_{2}$ & 2.33 & 2.26 & 2.29 & 13.97 & 13.54 & 13.75 \\
\hline $\mathrm{S}_{3}$ & 3.36 & 3.21 & 3.28 & 13.43 & 12.83 & 13.13 \\
\hline Mean & 2.44 & 2.33 & 2.38 & 13.49 & 12.83 & 13.16 \\
\hline $\mathrm{CD}_{0.05}$ & 0.08 & 0.08 & 0.08 & 0.51 & 0.43 & 0.47 \\
\hline \multicolumn{7}{|l|}{ Interaction: } \\
\hline $\mathrm{M}_{1} \mathrm{~S}_{1}$ & 1.25 & 1.18 & 1.22 & 10.03 & 9.43 & 9.75 \\
\hline $\mathbf{M}_{1} \mathbf{S}_{2}$ & 1.69 & 1.67 & 1.68 & 10.12 & 10.01 & 10.07 \\
\hline $\mathbf{M}_{1} \mathbf{S}_{3}$ & 2.52 & 2.37 & 2.44 & 10.07 & 9.46 & 9.77 \\
\hline $\mathbf{M}_{2} \mathbf{S}_{1}$ & 1.56 & 1.40 & 1.48 & 12.48 & 11.20 & 11.84 \\
\hline $\mathbf{M}_{2} \mathbf{S}_{2}$ & 2.19 & 1.97 & 2.08 & 13.15 & 11.82 & 12.49 \\
\hline $\mathbf{M}_{2} \mathbf{S}_{3}$ & 3.18 & 2.85 & 3.01 & 12.70 & 11.40 & 12.05 \\
\hline $\mathbf{M}_{3} \mathbf{S}_{1}$ & 1.85 & 1.74 & 1.79 & 14.77 & 13.89 & 14.33 \\
\hline $\mathbf{M}_{3} \mathbf{S}_{2}$ & 2.66 & 2.64 & 2.65 & 15.93 & 15.80 & 15.88 \\
\hline $\mathrm{M}_{3} \mathrm{~S}_{3}$ & 3.85 & 3.78 & 3.82 & 15.41 & 15.12 & 15.27 \\
\hline $\mathbf{M}_{4} \mathbf{S}_{1}$ & 1.88 & 1.74 & 1.81 & 15.04 & 13.91 & 14.48 \\
\hline $\mathbf{M}_{4} \mathbf{S}_{2}$ & 2.77 & 2.75 & 2.76 & 16.64 & 16.50 & 16.57 \\
\hline $\mathbf{M}_{4} \mathbf{S}_{3}$ & 3.89 & 3.83 & 3.86 & 15.55 & 15.30 & 15.43 \\
\hline Mean & 2.44 & 2.33 & 2.38 & 13.49 & 12.83 & 13.16 \\
\hline $\mathrm{CD}_{0.05}$ & 1.15 & 0.16 & 0.16 & NS & 0.86 & 0.94 \\
\hline
\end{tabular}

Table.5 Cost of cultivation of capsicum production as affected by different treatments

\begin{tabular}{|c|c|c|c|c|c|c|}
\hline $\begin{array}{l}\text { Sr. } \\
\text { No. }\end{array}$ & Treatment & $\begin{array}{c}\text { Fruit } \\
\text { yield } \\
\left(\mathrm{kg} / \mathrm{m}^{2}\right)\end{array}$ & $\begin{array}{c}\text { Gross returns } \\
\left(\text { Rs. } / \mathrm{m}^{2}\right)\end{array}$ & $\begin{array}{c}\text { Cost of } \\
\text { cultivation/ } \\
\text { treatment }\end{array}$ & $\begin{array}{c}\text { Net } \\
\text { returns } \\
\left(\text { Rs. } / \mathrm{m}^{2}\right)\end{array}$ & $\begin{array}{l}\text { Benefit-cost } \\
\text { ratio }\end{array}$ \\
\hline 1. & $\mathrm{M}_{1} \mathrm{~S}_{1}$ & 9.75 & 585.00 & 260.45 & 324.55 & 1.25 \\
\hline 2. & $\mathrm{M}_{1} \mathrm{~S}_{2}$ & 10.07 & 604.20 & 258.45 & 345.75 & 1.34 \\
\hline 3. & $\mathrm{M}_{1} \mathrm{~S}_{3}$ & 9.77 & 586.20 & 256.45 & 329.75 & 1.29 \\
\hline 4. & $\mathrm{M}_{2} \mathrm{~S}_{1}$ & 11.84 & 710.40 & 276.42 & 433.98 & 1.57 \\
\hline 5. & $\mathrm{M}_{2} \mathrm{~S}_{2}$ & 12.49 & 749.40 & 274.42 & 474.98 & 1.73 \\
\hline 6. & $\mathrm{M}_{2} \mathrm{~S}_{3}$ & 12.05 & 723.00 & 272.42 & 450.58 & 1.65 \\
\hline 7. & $\mathrm{M}_{3} \mathrm{~S}_{1}$ & 14.33 & 859.80 & 343.14 & 516.66 & 1.51 \\
\hline 8. & $\mathrm{M}_{3} \mathrm{~S}_{2}$ & 15.88 & 952.80 & 341.14 & 611.66 & 1.79 \\
\hline 9. & $\mathrm{M}_{3} \mathrm{~S}_{3}$ & 15.27 & 916.20 & 339.14 & 577.06 & 1.70 \\
\hline 10. & $\mathrm{M}_{4} \mathrm{~S}_{1}$ & 14.48 & 868.80 & 309.78 & 559.02 & 1.80 \\
\hline 11. & $\mathrm{M}_{4} \mathrm{~S}_{2}$ & 16.57 & 994.20 & 307.78 & 686.42 & 2.23 \\
\hline 12. & $\mathrm{M}_{4} \mathrm{~S}_{3}$ & 15.43 & 925.80 & 305.78 & 620.02 & 2.03 \\
\hline
\end{tabular}


A significant interaction between both the factors was recorded for yield per plant in $\mathrm{M}_{4} \mathrm{~S}_{3}$ and $\mathrm{M}_{4} \mathrm{~S}_{2}$ during both the years. The interaction of the $\mathrm{M}_{4}$ growing media with wider spacing of $45 \times 60 \mathrm{~cm}^{2}$ proved to be superior over the other treatment combinations. The comparison between $\mathrm{M}_{4} \mathrm{~S}_{3}$ and $\mathrm{M}_{4} \mathrm{~S}_{2}$ revealed that although the $\mathrm{M}_{4} \mathrm{~S}_{3}$ enhanced the growth and yield characters, the yield per $\mathrm{m}^{2}$ was higher at the $\mathrm{M}_{4} \mathrm{~S}_{2}(16.50$ $\mathrm{kg}$ ). This was probably due to the increase in the number of plants per unit area in $45 \times 45$ $\mathrm{cm}^{2}$ plant spacing, which might contribute to the extra yield per unit area leading to the high yield (Law-Ogboma and Egharevba, 2009).

\section{Benefit: Cost ratio}

The impact of growing media and plant spacing on benefit: cost ratio in capsicum cv. Orobelle under protected conditions has been worked. Therefore, for the economical analysis of various treatment combination of growing media and plant spacing the gross return, net return and $\mathrm{B}$ : $\mathrm{C}$ ratio have been studied. The relevant data is presented in Table 5. In the present investigation, maximum B: C ratio of 2.23:1 was obtained with combination $\mathrm{M}_{4} \mathrm{~S}_{2}$ (Soil + Cocopeat + Vermicompost + FYM (2:1:0.5:0.5) with plant spacing $45 \times 45 \mathrm{~cm}$ ), which was very close with the treatment combination $\mathrm{M}_{4} \mathrm{~S}_{3}$ (Soil + Cocopeat + Vermicompost + FYM (2:1:0.5:0.5) with plant spacing $45 \times 60 \mathrm{~cm})$ which obtained the B: C ratio of 2.03:1, whereas, minimum $\mathrm{B}$ : $\mathrm{C}$ ratio $(1.25: 1)$ was resulted from the treatment $\mathrm{M}_{1} \mathrm{~S}_{1}$ (Soil + Sand + FYM (2:1:1) with plant spacing $45 \times 30)$ $\mathrm{cm}$.

The result of this investigation showed that the growing media Soil + Cocopeat + Vermicompost + FYM (2:1:0.5:0.5) at a plant spacing $45 \times 60 \mathrm{~cm}^{2}$ and $45 \times 45 \mathrm{~cm}^{2}$ gave highest growth and yield characters of capsicum cv. Orobelle. It can be attributed to the better soil structure created by organic growing media, both physically and biologically, along with the constant and steady nutrient supply to the plants. Apart from this, the proper spacing led to the healthy competition amongst the plants giving superior results and made the cultivation of capsicum in polyhouse less tedious and more economical as well.

\section{References}

Bairwa, HL, Shukla AK, Mahawer LN, Kaushik RA, Shukla KB and Ameta KD. 2009. Response of integrated nutrient management on yield, quality and physico-chemical characteristics of okra cv. Arka Anamika. Indian J. Hortic., 66 (3)

Bisht BS 2012. Prospectus and Scenario of Protected Cultivation of Horticulture Crops in Uttrakhand. In: National seminar on Protected Cultivation of Vegetables and Flowers - a value addition chain. January 11-12, 2012 Pantnagar, pp1-8

Ganjare H, Futane N W, Dagwar S and Kurhade K. 2013. Growth and yield characters of capsicum in response to planting distance and sources of nutrients. Scholarly Journal of Agricultural Science 3(9): 386-390

Hala I, Larry M and Tsutomu O.2003. Effect of earthworm casts and compost on soil microbial activity and plant nutrient availability. Journal of Soil Biology and Biochemistry, 35: 295-302

Jaipual, Sharma S, Dixit A and Sharma AK. 2011. Growth and yield of capsicum (Capsicum annuum L.) and garden pea (Pisum sativum) as influenced by organic manures and biofertilizers. Indian Journal of Agricultural Science, 81(7): 637-642 
Kumar DM. 2011. Effect of planting geometry and foliar spray of micronutrients on plant growth, seed yield and quality of bell pepper (Capsicum annuиm L.) M.Sc. (Agri.) Thesis, University of Agricultral Science, Dharwad, Karnataka, India.

Kumar M and Kohli UK 2005. Capsicum production in naturally ventilated polyhouses in mid hills of Himachal Pradesh. In: International Conference on Plasticulture and Precision Farming, New Delhi on 17-21 Nov. pp 88

Law-Ogbomo K and Egharevba E. 2009. Effects of planting density and NPK fertilizer application on yield and yield components of tomato in forest location. World Journal of Agricultural Sciences, 5: $152-158$

Llaven MA, Jimenez JL, Coro BI, Rosales RR, Molina JM, Dendooven L, and Miceli FA. 2008. Fruit characteristics of bell pepper cultivated in sheep manure vermicompost substituted soil, Journal of Plant Nutrition, 31: 1585-1598

Malik AA, Chattoo MA, Sheemar G and Rashid R. 2011. Growth, yield and fruit quality of sweet pepper hybrid SH-SP-5 (Capsicum annuum L.) as affected by integration of in organic fertilizers and organic manures (FYM). Journal of Agricultural Technology, 7(4): 10371048
Mantur MS, Patil HB, Biradar. 2005. Productivity of capsicum in shade house as influenced by nutrition and planting geometry. In: International Conference on Plasticulture and Precision Farming, New Delhi. 92 Pp.

Natarajan S, Sasikala $\mathrm{S}$ and Kumaresan, GR. 2005. Influence of growing media, irrigation regime, nutrient management on growth, yield and economics of tomato under polyhouse condition. In:

International Conference on Plasticulture and Precision Farming, New Delhi on 17-21 Nov. pp 51

Roy S, Kumar N, Singh DK and Shrivastav AK. 2011. Effect of organic growing media and crop geometry on growth and yield of capsicum var. California wonder under protected condition in North West Himalayas. Vegetable Science, 38(1): 53-57

Sharma Sanjeev, Sharma AK, Jaipaul 2010. Performance of capsicum genotypes for horticultural traits and disease incidence under protected structure vis-a-vis open conditions. Indian J Hort., 67(4): 581583

Uma Maheswari T and Haripriya K. 2007. Comparative performance of hot pepper (Capsicum annuиm L.) cv. K2 with organic manures and inorganic fertilizers. Research on Crops, 8: 761764

\section{How to cite this article:}

Bijeta, Kuldeep Singh Thakur, Sandeep Kansal and Ashwini Kumar Sharma. 2018. Growth and Yield Characteristics of Capsicum (Capsicum annuиm L.) cv. Orobelle in Response to Different Growing Media and Plant Spacing under Protected Conditions. Int.J.Curr.Microbiol.App.Sci. 7(05): 1096-1103. doi: https://doi.org/10.20546/ijcmas.2018.705.134 